\title{
JOVIAN DECAMETRIC EMISSION WITH THE LONG WAVELENGTH ARRAY STATION 1 (LWA1)
}

\author{
T. E. Clarke*, C. A. Higgins ${ }^{\dagger}$, M. Imai ${ }^{\ddagger}$, and K. Imai ${ }^{\S}$
}

\begin{abstract}
The Long Wavelength Array Station 1 (LWA1) is located in central New Mexico, USA. It consists of 256 pairs of 'droopy-dipole' antennas operating between 10 and $88 \mathrm{MHz}$. The antennas are distributed in a pseudo-random layout across a $110 \mathrm{~m}$ $\times 100 \mathrm{~m}$ region. Observations with the LWA1 are based on peer reviewed proposals for open-skies observing time.

LWA1 is an excellent planetary radio emission instrument due to its sensitivity and the low radio frequency interference environment where it is located. We have undertaken several Jovian observing campaigns using the LWA1. We show that LWA1 data provide excellent spectral detail in Jovian decametric emission such as simultaneous left hand circular (LHC) and right hand circular (RHC) polarized Io-related arcs and source envelopes, modulation lane features, S-bursts structures, narrow band N-events, and apparent interactions between S-bursts and N-events. The start of the LHC Io-C source region was traced to earlier longitudes than typically found in the literature. Early LWA1 observations revealed a wealth of Io-D emission, including detection of rare S-bursts during an Io-D event.

These initial results have led to new programs to explore the spectral characteristics of Io-D events, investigate modulation lanes of Io-B/Io-C events and examine the beaming structure of S-bursts combining LWA1, NDA, and URAN2. In addition, LWA1 is one of the ground-based support facilities for the NASA JUNO ${ }^{1}$ mission.
\end{abstract}

\section{Introduction}

The connection between strong bursts of radio emission below $40 \mathrm{MHz}$ and the planet Jupiter was identified by Burke and Franklin [1955]. Ground-based observations show

\footnotetext{
* Remote Sensing Division, US Naval Research Laboratory, Washington, DC, USA

${ }^{\dagger}$ Department of Physics and Astronomy, Middle Tennessee State University, Murfreesboro, TN, USA

$¥$ Department of Physics and Astronomy, University of Iowa, Iowa City, IA, USA

$\S$ Department of Electrical Engineering and Information Sci., Kochi National College of Technology, Kochi, Japan

${ }^{1}$ https://www.nasa.gov/mission \_pages/juno/main/index.html
} 
that the emission can be traced from the ionospheric cutoff ( $>5 \mathrm{MHz}$ ) to $39.5 \mathrm{MHz}$ with the peak of the spectral power at a frequency of about $10 \mathrm{MHz}$. The peak flux of the emission is $>10^{-19} \mathrm{~W} / \mathrm{m}^{2} / \mathrm{Hz}$ (with short timescale S-bursts exceeding this flux by factors of 100).

Both ground-based and space-based observations have revealed that Jupiter decametric (DAM) emission is correlated with auroral magnetic field lines (non-Io related emission) as well as with magnetic field lines near Io and the Io plasma torus (Io-related emission), see review by Carr et al. [1983]. These coherent emissions are driven by wave-particle interactions near the local electron cyclotron frequency, called the cyclotron maser instability (CMI) mechanism [Wu and Lee, 1979].

The electrons gyrate along high latitude magnetic field lines, and when the local plasma density is low such that the plasma freq/cyclotron freq $<<1$, the keV electrons couple with the electric field resulting in wave amplification. The waves are generated in both the ordinary $(\mathrm{O})$ mode and extraordinary $(\mathrm{X})$ mode, with the X-mode dominating, in theory, by many orders of magnitude [Melrose et al., 1984]. Due to the beaming geometry, right-hand circularly $(\mathrm{RHC})$ polarized waves are generated from the northern hemisphere sources while left-hand circularly (LHC) polarized waves come from the southern hemisphere. Northern hemisphere Io-controlled emission is called Io-A and Io-B for the trailing and leading edges of the emission cone, while the trailing and leading edges are named Io-C and Io-D for southern hemisphere emission. Detailed study of the complex Jovian emission requires an instrument with excellent full polarization capability, frequency coverage down to the ionospheric cutoff and a low radio frequency interference (RFI) environment.

Jovian emission is characterized by variations on a wide range of temporal scales. In this paper, we present an overview of long decametric storms in Section 3.1 which cover timescales of up to several hours, while in Section 3.2 we present fine temporal scale structures such as modulation lanes which last several minutes, long bursts, or L-bursts, which have timescales of several seconds, and short bursts, or S-bursts, which have timescales of several milliseconds.

We have used the powerful new Long Wavelength Array Station 1 (hereafter LWA1) to undertake high temporal and spectral resolution ground-based studies of the Io-driven Jovian emission. These observations have shown the LWA1 to be an excellent new tool to help refine the details of the decametric emission.

\section{The Long Wavelength Array Station 1}

The Long Wavelength Array Station 1 (Figure 1) is located in central New Mexico, USA. This single station contains 256 pairs of 'droopy-dipole' antennas operating between 10 and $88 \mathrm{MHz}$. LWA1 antennas are designed to have $6 \mathrm{~dB}$ gain above the galactic background over the majority of the observational band. A detailed description of the LWA1 antennas is presented in Hicks et al. [2012].

The LWA1 station antennas are distributed in a pseudo-random layout across a $110 \mathrm{~m}$ by $100 \mathrm{~m}$ region. The station is located near the core of the National Radio Astronomy 


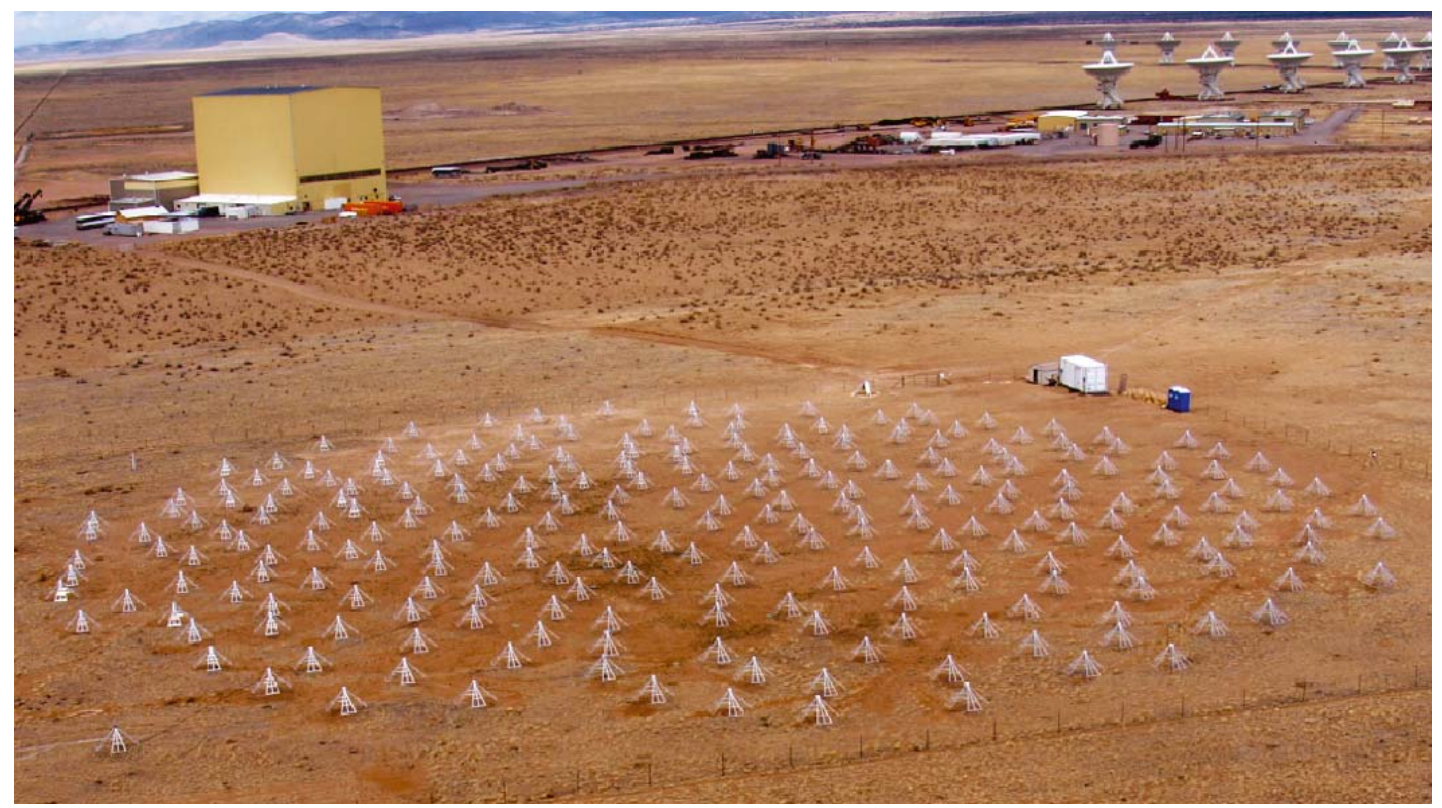

Figure 1: The 256 dual-polarization dipoles of the LWA1 are seen in the foreground with the associated electronics shelter to the right of the station and the National Radio Astronomy Observatory's Jansky Very Large Array in the background (reproduced with permission from Greg Taylor).

Observatory's Karl G. Jansky Very Large Array. The radio frequency interference environment at the LWA1 site is very low and the instrument can regularly observe down to $11 \mathrm{MHz}$ at nighttime.

The station is able to record data in beamformed mode with 4 independent, dual polarization beams or in transient buffer mode with a short timescale wideband mode or a continuous narrow-band mode. Jovian data presented in this paper was recorded in beamformer mode as raw voltages. We note that LWA1 also has a spectrometer mode which provides lower spectral and temporal resolution data. A more complete description of the LWA1 is presented in Ellingson et al. [2013] and Taylor et al. [2012].

In Figure 2 we show a plot of the sensitivity of LWA1 compared to other a number of instruments used for Jovian study. The sensitivities have been calculated assuming a one hour observation at zenith and a bandwidth of $50 \mathrm{kHz}$ for all instruments. Sensitivities were determined using effective areas for each instrument as follows: University of Florida ${ }^{2}$ (UFRO) Tee Pee and Yagi, Low Frequency Array (LOFAR, van Haarlem et al. [2013]), Nançay Decametric Array (NDA, Boischot et al. [1980]), Long Wavelength Array Station 1 (LWA1, Taylor et al. [2012]), Ukrainian Radio Interferometer of NASU-2 (URAN2, Brazhenko et al. [2005]), and Ukrainian T-shaped Radio telescope, second modification (UTR-2, Konovalenko et al. [2016]). While we recognize that these observing parameters are not ideal for Jovian studies and in particular the bandwidth does not accurately represent the instantaneous capabilities of the instruments, we feel that it is necessary to impose these conditions to make a fair comparison. We further note that we show

\footnotetext{
${ }^{2}$ http://ufro1.astro.ufl.edu/observatory.htm
} 
sensitivities plotted from the lowest observing frequency of each instrument up to a cutoff of $40 \mathrm{MHz}$ as appropriate for Jovian studies.

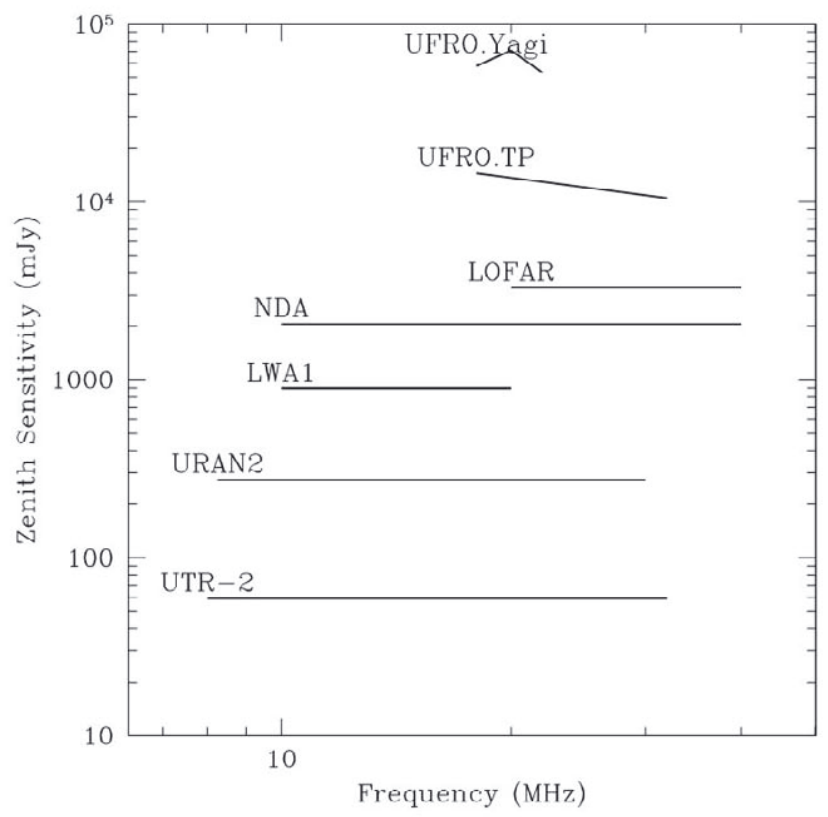

Figure 2: Plot of instruments sensitivity vs frequency for LWA1 in comparison to UFRO, UTR-2 URAN2, NDA and LOFAR. All sensitivities are calculated assuming a $50 \mathrm{kHz}$ instantaneous bandwidth and 1 hour integration at zenith. Sensitivities are plotted from the lowest observing frequency of each instrument to a cutoff of $40 \mathrm{MHz}$ as appropriate for Jovian studies.

LWA1 is an excellent planetary radio emission instrument due to its sensitivity, polarization capability, and the low radio frequency interference environment. We have undertaken several Jovian observing campaigns using the LWA1. Observations have been primarily recorded in raw data beamforming mode with two overlapping tunings of bandwidth $16 \mathrm{MHz}$ per tuning, allowing observations using a single beam to instantaneously cover the frequency range of 10-40 MHz. Throughout this work we produce spectrograms with frequency resolution of up to $5 \mathrm{kHz}$ and temporal resolution of up to 0.21 milliseconds.

\section{Jovian observations}

It has long been recognized that Io-controlled Jovian emission occupies characteristic parameter space in a plot of Io phase versus central meridian longitude (CMLIII) [e.g. Carr et al., 1983]. Using this occurrence probability plot, we targeted Io-driven emission that passed through high elevation for LWA1 observations as described in Clarke et al. [2014].

Targeted observations of Jovian emission with the LWA1 began in 2012. In Table 1 we summarize the major Jovian observing programs which have been run on the instrument through the end of the sixth Call for Proposals (CFP) in late 2016. 
Table 1: Table of LWA1 Jovian observing programs

\begin{tabular}{|c|c|c|c|c|}
\hline ID & PI & $\begin{array}{c}\text { Start/End Date } \\
\text { (YY-MM-DD) }\end{array}$ & Hours & Science Goal \\
\hline LC001 & Clarke & $12-09-11 / 12-11-06$ & 107 & Io-A/C \& Io-B fine structure \\
LH004 & Higgins & $12-09-23 / 13-12-31$ & 136 & Io-related polarimetry \\
LH010 & Higgins & $13-10-05 / 14-08-28$ & 156 & Io-D fine structure \\
LH013 & Higgins & $14-12-12 / 15-03-13$ & 237 & Io-B fine structure/ \\
& & & & modulation lanes \\
LR004 & Romero-Wolf & $15-01-26 / 15-03-04$ & 5 & Jovian moon reflections \\
LH014 & Higgins & $16-01-22 /{ }^{3}$ & 237 & JUNO mission support \\
LI001 & M. Imai & $16-01-08 / 16-08-05$ & 29 & LWA1-NDA-URAN2 S-bursts \\
LR005 & Romero-Wolf & $15-12-20 / 16-05-29$ & 27 & Jovian moon reflections \\
\hline
\end{tabular}

Observations undertaken for the programs presented in this paper were designed to allow characterization of the full event

Observing sessions varied in length from one to five hours, thus the source elevation could undergo significant change over the course of the observation. Due to the nature of dipole arrays, the polarization must be carefully characterized off zenith to correct for polarization leakage across Stokes parameters. For the observing campaigns presented here such characterization was not yet available. We thus present only analysis of the overall emission structure as well as fine structure and the handedness of the emission. Future work will concentrate on the polarization properties of the Jovian emission.

In the remainder of this section we briefly summarize a few examples of LWA1 observations from the first two Jovian campaigns (LC001 and LH004).

\subsection{Overall emission structure}

Raw voltages were recorded and then converted to frequency-time spectrograms to examine the emission structure. Initial low temporal and spectral resolution (2 seconds and $10 \mathrm{kHz}$, respectively) spectrograms were used to characterize the overall structure of the emission.

In Figure 3 we show the low temporal and spectral resolution spectrogram of an Io-A/Io$\mathrm{C}$ event recorded on December 01, 2012. Right hand circular emission (RHC) is shown in black while the left hand circular emission (LHC) is shown in red. The RHC Io-A emission shows the typical vertex late arc structures associated with the trailing edge of the emission cone. The Io-A emission is followed at CMLIII of $270^{\circ}$ by a second RHC 'great arc' [see e.g. Boudjada et al., 1995]. A LHC Io-C emission component is detected starting at a CMLIII of $230^{\circ}$ near $11 \mathrm{MHz}$, peaking at a maximum frequency of $24.5 \mathrm{MHz}$ around CMLIII of $295^{\circ}$ and dropping below the LWA1 observing band around CMLIII of $350^{\circ}$. This Io-C event begins well before the nominal CMLIII of $280^{\circ}$ defined in Carr et

$\overline{{ }^{3} \text { Observations continue at time of writing. }}$. 


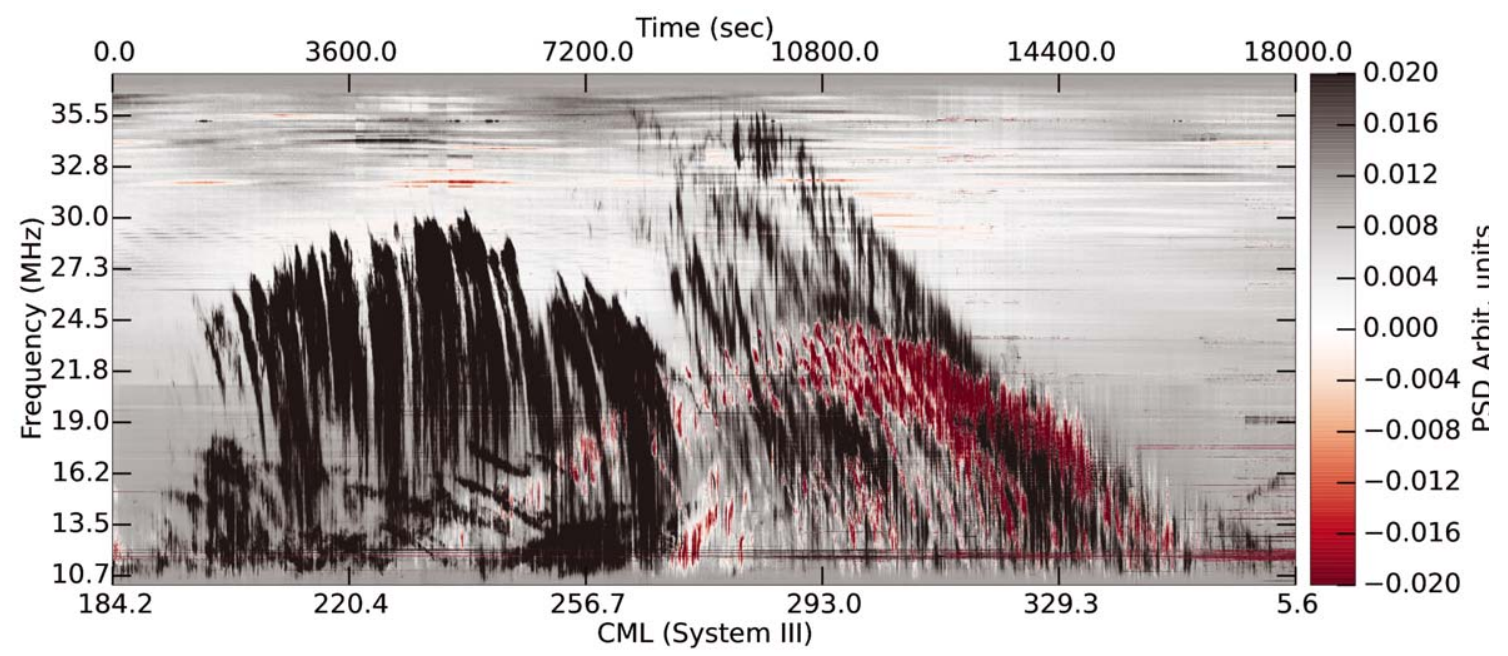

Figure 3: Five hour LWA1 observation of Io-A/Io-C event from December 01, 2012 shown with temporal resolution of 2 seconds and spectral resolution of $10 \mathrm{kHz}$. Upper axis shows time since observation start in seconds while lower axis shows CMLIII. Note that frequencies increase from the bottom to the top of the plot. The color scale shows the power spectral density with black being right hand circular and red being left hand circular (from Clarke et al. [2014]).

al. [1983] and highlights the need to consider a frequency-dependent CMLIII range for storms.

Emission from the leading edge of emission cones is seen in Figure 4 where we show a two hour spectrogram for an Io-B event on December 27, 2012. This event was unusual in the fact that the emission is seen to cut off sharply near CMLIII $=121^{\circ}$. It is likely that the Io-B emission cone rotated out of the line of sight to Earth at this point causing the abrupt end to the emission. The loss of a direct line of sight to the emission cone is supported by the Io-phase vs CMLIII probability plot where the loss of emission coincides with the CMLIII where the observation passes outside the high probability region. At roughly the same time as the Io-B emission ends, we observed LHC Io-D emission enter our observing window at the lowest observing frequencies.

Jovian decametric emission is highly elliptically polarized and will undergo Faraday rotation as it passes through a magnetized, ionized medium such as the Io plasma torus and Earth's ionosphere. In Figure 5 we show Faraday fringes in the linear polarization difference spectrogram for the same Io-B/Io-D event seen in Figure 4. Note the near horizontal Faraday fringes visible in the data. The observed spacing of the fringes decreases with decreasing frequency. Using the nulls of the fringes, we measured a rotation measure of $2.2 \mathrm{rad} / \mathrm{m}^{2}$ which is primarily dominated by Earth's ionosphere.

\section{$3.2 \quad$ Fine structure}

Jovian decametric emission shows a wide range of fine structures including short and longer time scale modulation. 


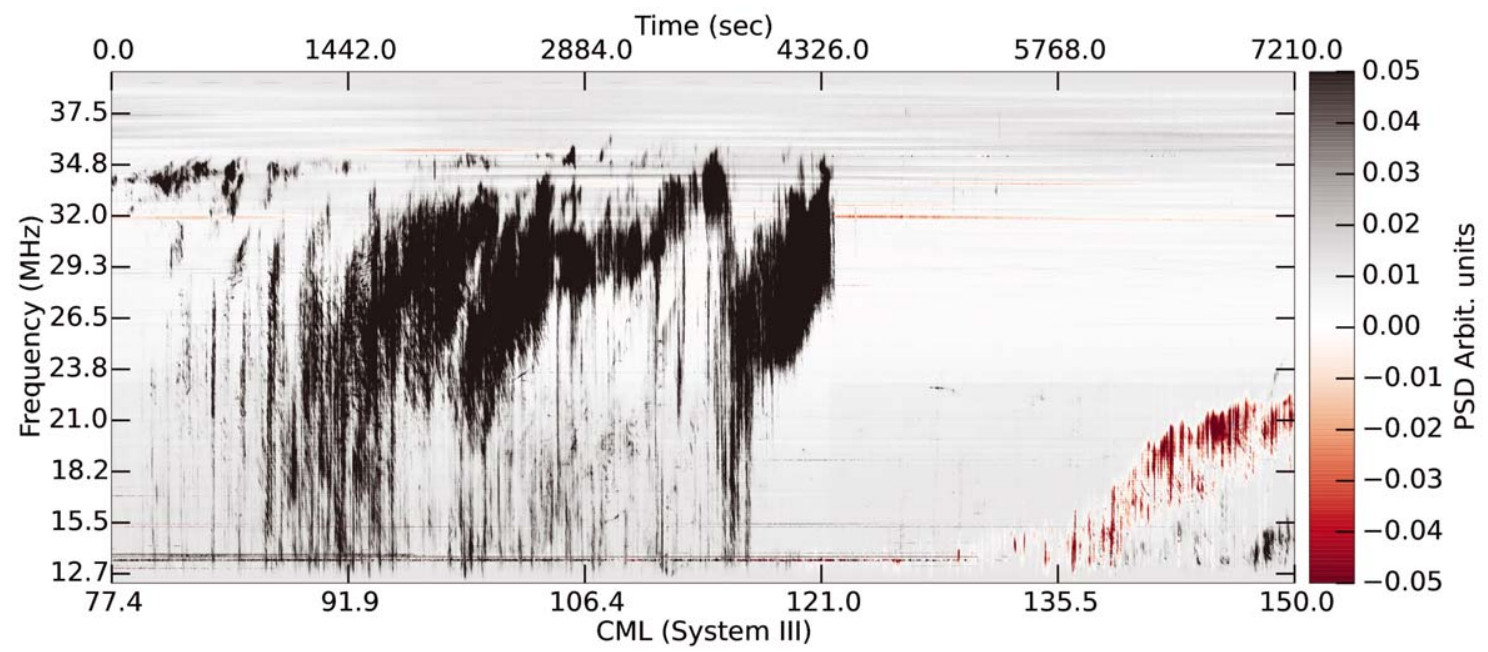

Figure 4: Two hour LWA1 observation of Io-B/Io-D event from December 27, 2012 with temporal resolution of 2 seconds and spectral resolution of $10 \mathrm{kHz}$. Axes similar to Figure 3 (from Clarke et al. [2014]).

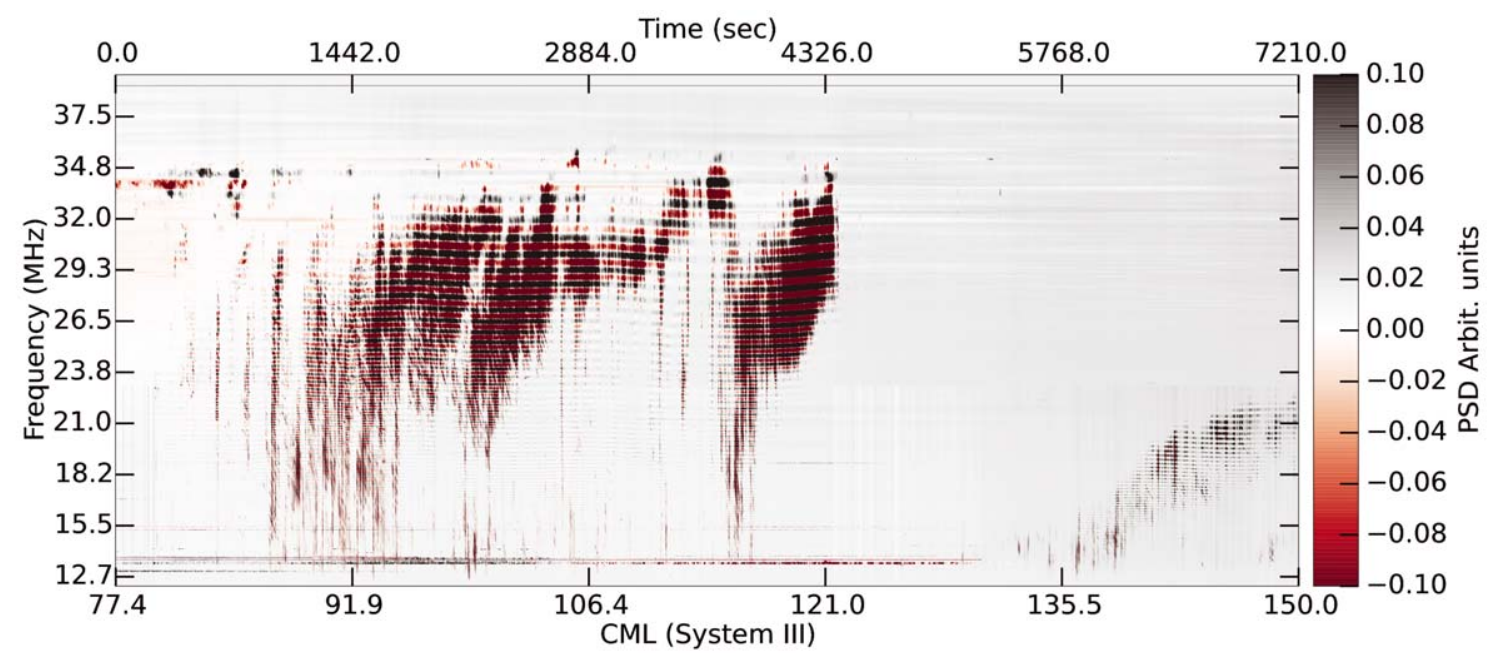

Figure 5: Faraday rotation for Io-B/Io-D event from December 27, 2012. Axes similar to Figure 3 (from Clarke et al. [2014]).

In Figure 6 we show modulation lanes across a portion of the Io-B emission from the event shown in Figure 4. The modulation lanes are seen as a series of nested narrow, curved lines that sweep from low frequency to high frequency as time passes, cutting across the bright L-burst emission structures seen in the Jovian emission. These modulation lanes, originally identified by Riihimaa [1968], are characterized by a strong periodicity with time and can have either positive or negative slopes. Measured slopes of the modulation lanes in the 21-23 MHz portion of the LWA1 spectrum are consistent with those of Riihimaa [1968]. A model to explain the production of the modulation lanes was developed by Imai et al. [1997; 2002; 2006]. By using this model, Jupiter's radio source locations and 


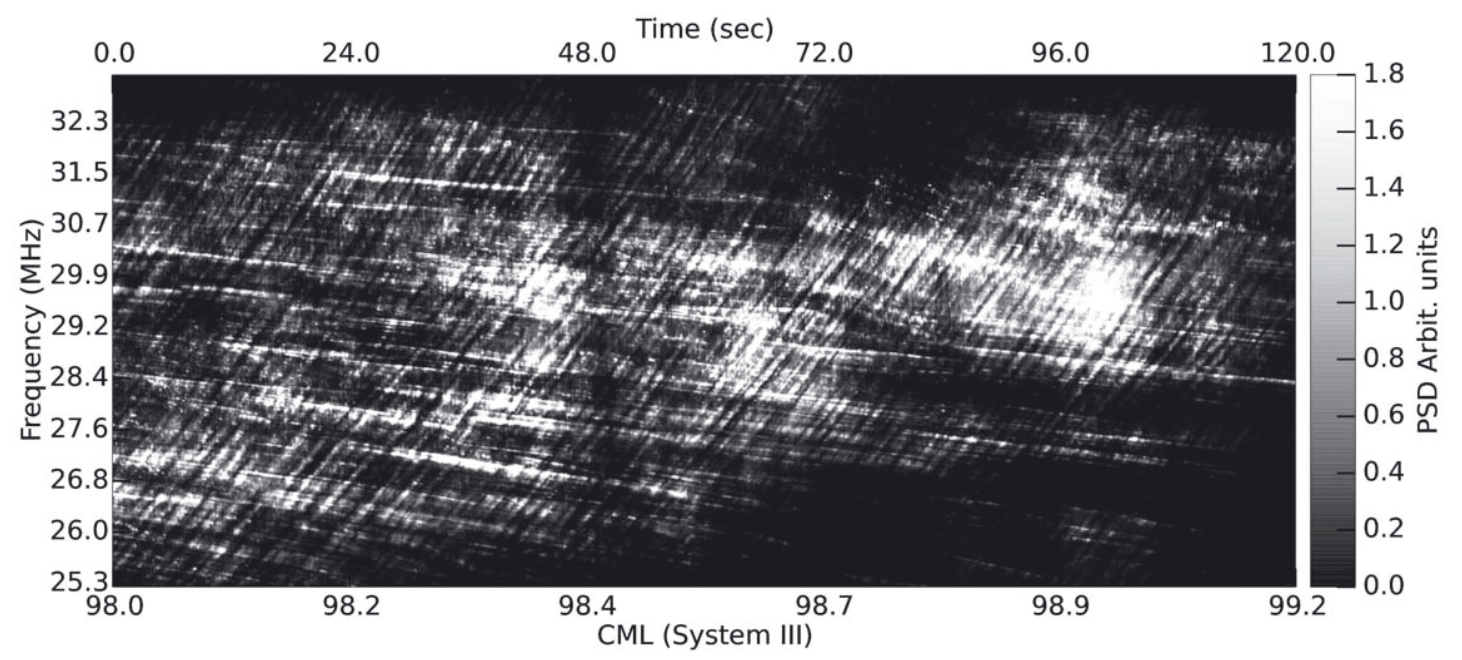

Figure 6: Modulation lanes showing positive slope over a portion of the December 27, 2012 Io-B event from Figure 4. This image has a temporal resolution of $10 \mathrm{~ms}$ and a spectral resolution of $10 \mathrm{kHz}$ (from Clarke et al. [2014]).

beam parameters can be measured. This remote sensing tool is called the modulation lane method.

Rapid bursts of emission seen in the complex Jovian decametric observations are labeled as short bursts or S-bursts. These short pulses of emission are characterized by timescales in the range of 1 to $100 \mathrm{~ms}$. The emission is confined to a narrow instantaneous frequency band with the burst frequency drifting with time. Negative drift rates are thought to be the result of the motion of the packets of radiating electrons outward along Jovian magnetic field lines to regions of lower magnetic field strength. We refer the reader to detailed discussions of S bursts presented in Carr et al. [1983] and Zarka et al. [1996]. In Figure 7 we show a high spectral $(10 \mathrm{kHz})$ and temporal $(0.25 \mathrm{~ms})$ resolution spectrogram with S bursts from a small portion of the December 27, 2012 event shown in Figure 4. These S-bursts are observed in the 15-18 MHz frequency range and have measured drift rates of -11 to $-15 \mathrm{MHz} / \mathrm{s}$. These LWA1 observations of drift rates of S-bursts for Io$\mathrm{D}$ events are potentially very important since there is little information available in the literature on the detection and drift rate of S-bursts during Io-D events.

Detailed LWA1 study of an Io-B event recorded on March 11, 2012 revealed that the portion of the spectrum between 21 and $25 \mathrm{MHz}$ was largely dominated by S-bursts while the lower frequency portion of the spectrum (below $21 \mathrm{MHz}$ ) was a mix of S-bursts and narrow frequency bands, or N-events. N-events are relatively rare and consist of narrow frequency bands (200-600 kHz wide) of emission that can drift up or down over time. The bursts last for extended times over several seconds to tens of minutes. These events are described in detail in Riihimaa [1985]. In Carr et al. [1983] and Riihimaa and Carr [1981] there are discussions of the interaction of N-events with S-bursts.

In Figure 8 we show a 200 ms portion of the March 11, 2012 event from LWA1 observations using a spectral resolution of $10 \mathrm{kHz}$ and a temporal resolution of $0.25 \mathrm{~ms}$. This figure shows an S-burst that starts near a frequency of $25.6 \mathrm{MHz}$ with a drift rate of $-17.9 \mathrm{MHz} / \mathrm{s}$. 


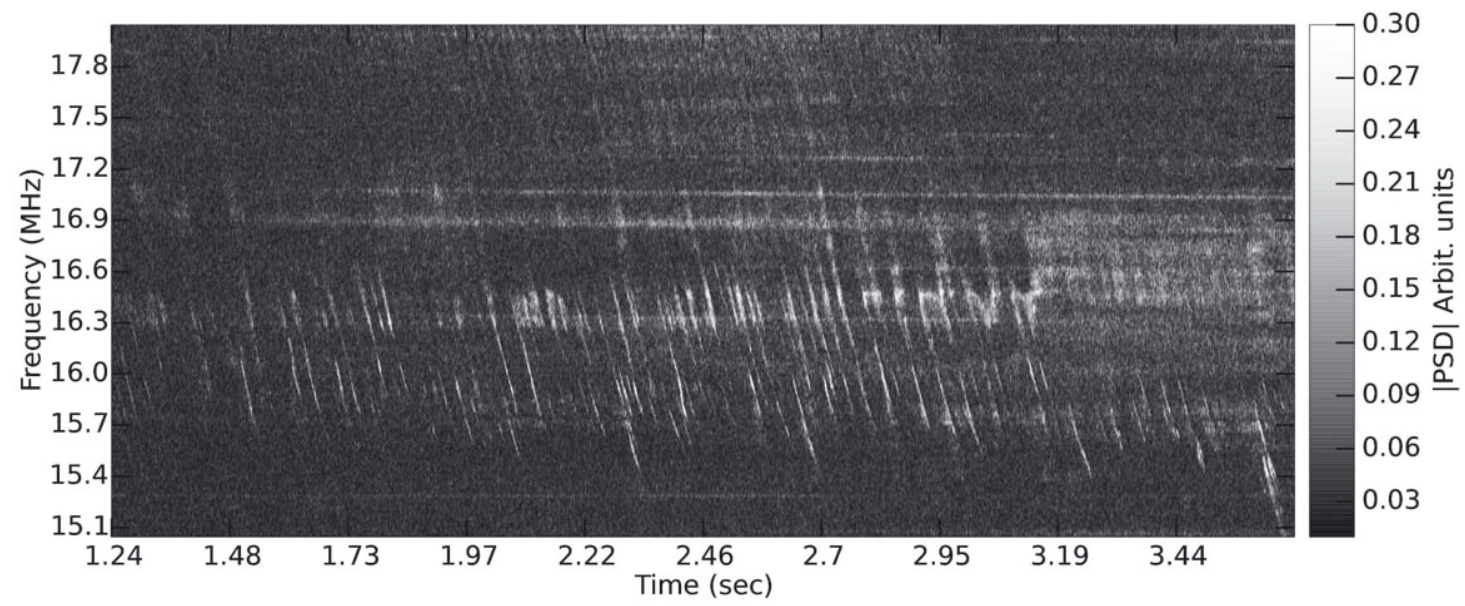

Figure 7: S-bursts for a short portion of the December 27, 2012 Io-D event shown in Figure 4 with a temporal resolution of $0.25 \mathrm{~ms}$ and spectral resolution of $10 \mathrm{kHz}$. These short time scale bursts are seen in the LHC emission. The figure starts 6236 seconds into the event.

The burst drifts downward to a frequency of $23.7 \mathrm{MHz}$ where the spectrogram shows the S-burst intersects in frequency-time space with a narrow-band region of emission that is roughly constant in frequency. The narrow-band region is similar to longer N-events but only commences $15 \mathrm{~ms}$ prior to the frequency-time interaction with the S-burst. At the frequency-time intersection with the S-burst, both bursts end abruptly. Following this apparent quenching, there appear to be multiple S-bursts generated to lower frequencies with drift rates of $-16.3 \mathrm{MHz} / \mathrm{s}$. There are numerous examples of similar 'interactions' in this region of the spectrum which suggest the possibility that these are not coincidental connections but that the source regions for the S-bursts and narrow-band may be cospatial.

Interactions between S-bursts and N-events have been reported in the literature [Oya et al. 2002; Riihimaa and Carr, 1981] and are often characterized by an inverted tilted V spectral shape connecting the S-burst and N-event. An example of such a connection is seen in Figure 9 where we show a 4.3 second spectrogram for an Io-B event recorded by the LWA1 on September 24, 2012. This event likely consists of a combination of L-bursts and S-bursts, but also includes N-event signatures. The wispy L-bursts and the stronger N-events are both interrupted by the S-bursts, causing the shadow to lower frequency (i.e. below) the inverted $\mathrm{V}$ structure of the S-bursts. These events are similar to, but faster than, the slow-drift shadow events described in Koshida et al. [2010].

The origin of these events is not understood although there is the suggestion that they may be the result of bunched electrons accelerated upward by electric fields along the Io flux tube [Oya et al., 2002]. These interactions (together with other events presented in this paper) demonstrate the excellent sensitivity and resolution of the LWA1 instruments. More observations are needed to fully characterize and understand the complex Jovian emission structures. 


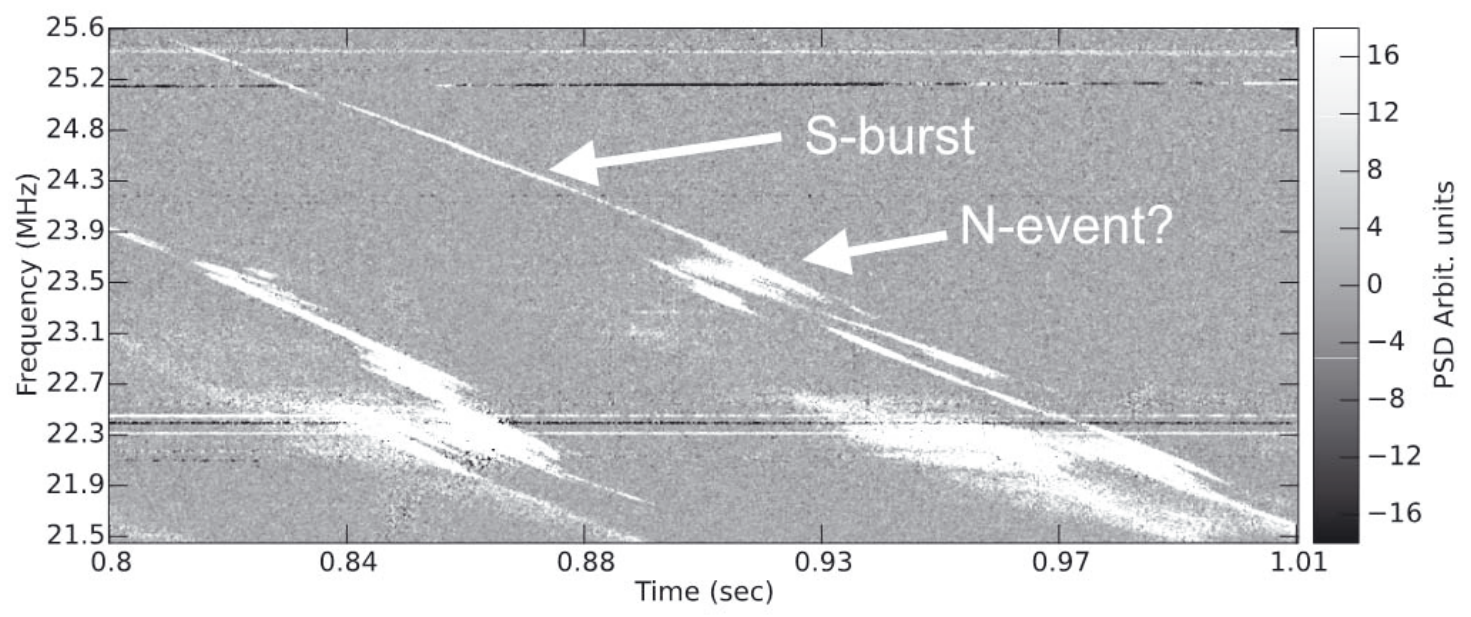

Figure 8: High temporal and spectral resolution (0.25 ms and $10 \mathrm{kHz})$ LWA1 observation of a portion of an Io-B event from March 11, 2012 showing the potential interaction of an S-burst and narrow-band event. We label the narrow-band event with a question mark in recognition of the fact that it is much shorter than classical N-events.

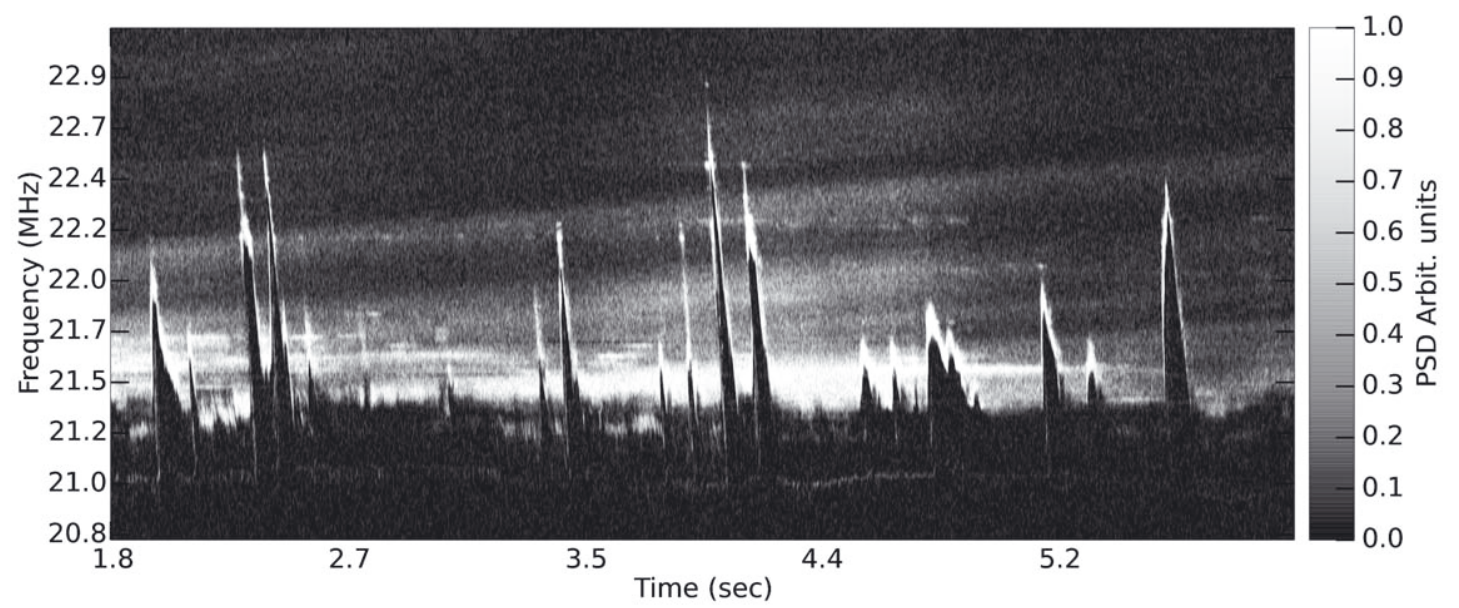

Figure 9: LWA1 spectrogram of an Io-B event from September 24, 2012 showing complex structure consisting of L-bursts and S-bursts as well as $\mathrm{N}$-event. The spectrogram demonstrates the tilted- $V$ shaped narrow bands of emission with shadow events devoid of emission at frequencies below the tilted $V$ shapes (from Clarke et al. [2014]).

\section{S-burst beaming mechanism}

Two proposed beaming mechanisms for the S-bursts are flashlight-like beaming where the radio sources associated with the S-bursts at Jupiter result in isotropic emission toward the observers, and the beacon-like beaming model where the emitting sources are confined to narrow longitude ranges which rotate with Jupiter about the magnetic axis. The two primary emitting models can be distinguished through simultaneous measurements of 
time lags of common S-bursts observed from radio telescopes separated by long baselines at Earth.

We have made simultaneous observations with the LWA1, the Nançay Decameter Array [Boischot et al., 1980; Lecacheux, 2000, NDA, in Nançay, France], and URAN2 [Brazhenko et al., 2005, located in Poltava, Ukraine] to undertake a cross-correlation analysis of Sbursts over baselines up to $8640 \mathrm{~km}$ [Imai et al., 2016]. Using 105 minutes of data, we have measured time lags of S-bursts using a cross-correlation analysis of the spectrograms from all three instruments. In Figure 10 we show the mean cross-correlation functions for the instruments together with the predicted delays from the two emission models and the typical delay errors for the instrument pairs. These plots show that the flashlightlike beaming model fits well to the observed values. Based on these observations, Imai et al. [2016] were further able to conclude that the S-burst has a minimum beaming (cone) thickness of 2.66" (i.e., the angular size estimated from the maximum baseline at LWA1-URAN2) in the east-west direction projected on Jupiter.

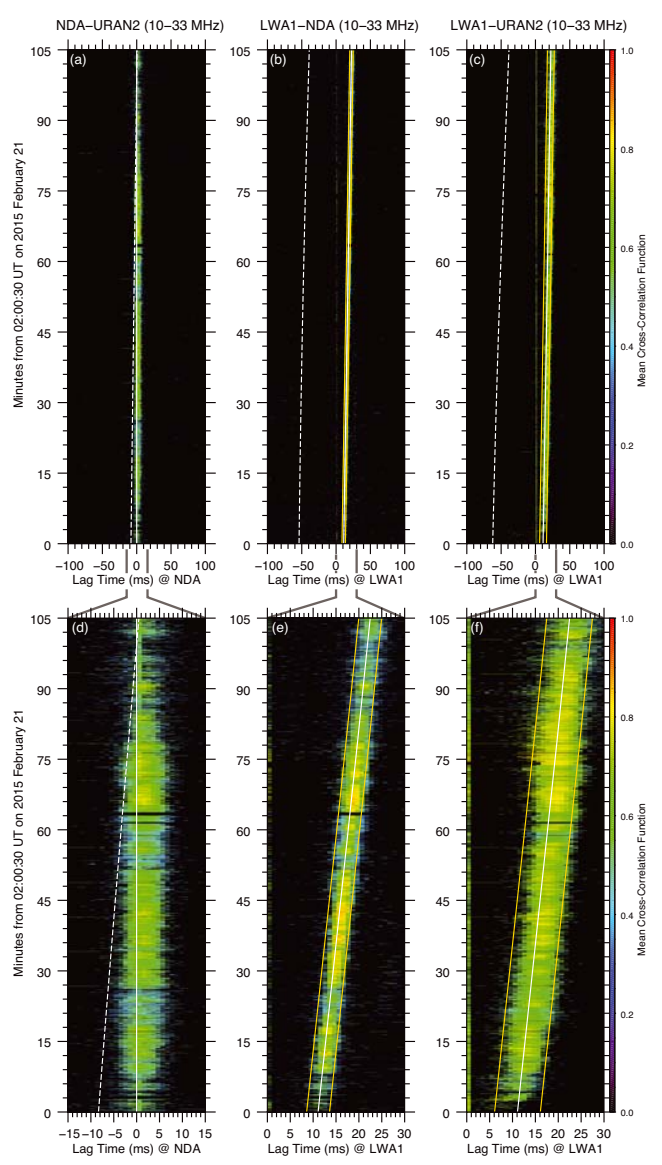

Figure 10: Instrument mean cross-correlation functions between 10.2 and $33.0 \mathrm{MHz}$ for $\mathrm{NDA}-$ URAN2 (plot a,d), LWA1-NDA (plots b,e), and LWA1-URAN2 (plots c,f). White solid line is the expected delay from the flashlight-like model and the white dashed line is the delay expected for the beacon-like model. Yellow lines are the $2.5 \mathrm{~ms}$ errors for the LWA1-NDA pair and $5 \mathrm{~ms}$ for the LWA1-URAN2 pair. Figure taken from Imai et al. [2016], reproduced by permission of the $A A S$. 


\section{Summary}

We have shown several examples of the scientific impact of the Long Wavelength Array Station 1 instrument on Jovian decametric emission studies. Analysis of data from our initial observing campaign revealed details of Io-C emission occurring simultaneously with Io-A emission. We have shown that LWA1 is able to trace the starting CMLIII range for Io-C emission down to frequencies near $11 \mathrm{MHz}$. The excellent polarization capabilities of the LWA1 allow us to investigate the Faraday rotation of the Jovian emission which is a mix of a dominant contribution from Earth's ionosphere and minor components from near the Jovian emission source and the Io plasma torus. We have also studied the fine-scale structure of the Jovian emission, including modulation lanes and S-bursts.

LWA1 observations clearly revealed the presence of modulation lanes across all Io-related emission sources. Analysis of the drift rates of the modulation lanes show that they are consistent with results in the literature. A more detailed analysis of a broad set of modulation lanes from LWA1 is presented by K. Imai et al. [2017].

Our observations clearly revealed the fine-scale structure of S-bursts, N-events, S-N events and tilted-V events. Several N-events were seen within the data for Io-A, Io-B, and Io$\mathrm{D}$ events. We also identified long trains of events that included S-N-events where there appear to be complex interactions between the S-burst and N-event.

Left hand circular emission associated with Io-D sources was clearly seen in the lower observing frequencies. Due to the low RFI environment at the low frequency end of our observing band, we were able to study the fine scale structure S-bursts seen within the Io-D emission. Recent LWA1 observations of Jupiter DAM show that the Io-D radio source has ubiquitous modulation lane structures, S-bursts, and narrow band events, and that the Io-D source is seen at a broader CML and Io phase range [see Higgins et al., 2017]. These features, some not seen before, are due to the excellent spectral and temporal resolution of the LWA1 instrument in addition to full Stokes information.

Finally, we note that our recent Jovian studies have included S-burst beaming measurements using LWA1, Nançay Decameter Array, and URAN2.

The LWA1 Jupiter data at the University of Iowa das2 server (http://das2.org) are accessible at http://www-pw.physics.uiowa.edu/earth/ and these data can be displayed with the Autoplot interactive browser. LWA1 is currently providing ground-based observing supporting to the NASA Juno mission from January 2016 through the time of this writing. This included the Juno perijove 3 in December of 2016 and perijove 4 in January-February 2017.

Acknowledgments. Basic research in Radio Astronomy at the US Naval Research Laboratory is supported by 6.1 Base Funding. This research has been supported in part by JSPS KAKENHI grant 16K05570. Construction of the Long Wavelength Array Station 1 (LWA1) was supported by the Office of Naval Research under contract N00014-07-C-0147. Support for operations and continuing development of LWA1 is provided by the National Science Foundation under grants AST-1139963 and AST-1139974 of the University Radio Observatories program. The Editors thank V. Ryabov and one anonymous reviewer for their help in evaluating this paper. 


\section{References}

Boischot, A., C. Rosolen, M. G. Aubier, G. Daigne, F. Genova, Y. Leblanc, A. Lecacheux, J. de la Noë, and B. M. Pedersen, A new high gain, broadband steerable, array to study Jovian decametric emission, Icarus, 43, 399-407, 1980.

Boudjada, M. Y., H. O. Rucker, and H. P. Ladreiter, The Io-C Jovian decameter emissions, Astron. Astrophys., 303, 255-264, 1995.

Brazhenko, A. I., V. G. Bulatsen, R. V. Vashchishin, A. V. Frantsuzenko, A. A. Konovalenko, I. S. Falkovich, E. P. Abranin, O. M. Ulyanov, V. V. Zakharenko, A. Lecacheux, and H. O. Rucker, New decameter radiopolarimeter URAN-2, Kinematika i Fizika Nebesnykh Tel Suppl., 5, 43-46, 2005.

Burke, B. F., and K. L. Franklin, Radio emission from Jupiter, Nature, 175, p.1074, 1955.

Carr, T.D., M.D. Desch, and J.K. Alexander, Phenomenology of magnetospheric radio emissions, in Physics of the Jovian Magnetosphere, edited by A. J. Dessler, Cambridge University Press, New York, USA, 226-284, 1983.

Clarke, T. E., C. A. Higgins, J. Skarda, K. Imai, M. Imai, F. Reyes, J. Thieman, T. Jaeger, H. Schmitt, N.P. Dalal, J. Dowell, S. W. Ellingson, B. Hicks, F. Schinzel, and G. B. Taylor, Probing Jovian decametric emission with the Long Wavelength Array station 1, J. Geophys. Res., 119, 9508-9526, 2014.

Ellingson, S. W., G. B. Taylor, J. Craig, J. Hartman, J. Dowell, C. N. Wolfe, T. E. Clarke, B. C. Hicks, N. E. Kassim, P. S. Ray, L. J. Rickard, F. K. Schinzel, and K. W. Weiler, The LWA1 radio telescope, IEEE Transactions on Antennas and Propagation, 61, 2540-2549, 2013.

Hicks, B. C., N. Paravastu-Dalal, K. P. Stewart, W. C. Erickson, P. S. Ray, N. E. Kassim, S. Burns, T. Clarke, H. Schmitt, J. Craig, J. Hartman, and K. W. Weiler, A wideband, active antenna system for long wavelength radio astronomy, Publ. Astron. Soc. Pacific., 124, 1090-1104, 2012.

Higgins, C., T. E. Clarke, K. Imai, M. Imai, F. Reyes, and J. Thieman, Morphology of the Jupiter Io-D decametric radio source, in Planetary Radio Emissions VIII, edited by G. Fischer, G. Mann, M. Panchenko, and P. Zarka, Austrian Academy of Sciences Press, Vienna, 77-88, 2017.

Imai, K., L. Wang, and T.D. Carr, Modeling Jupiter's decametric modulation lanes, J. Geophys. Res., 102, 7127-7136, 1997.

Imai, K., J. J. Riihimaa, F. Reyes, and T. D. Carr, Measurement of Jupiter's decametric radio source parameters by the modulation lane method, J. Geophys. Res., 107, A6, id.1081, 2002.

Imai, K., F. Reyes, T. D. Carr, and A. Lecacheux, Recent progress in the measurement of Jupiter's decametric radio source parameters by the modulation lane method, in Planetary Radio Emissions VI, edited by H. O. Rucker, W. S. Kurth, and G. Mann, Austrian Academy of Sciences Press, Vienna, 213-221, 2006. 
Imai, K., C. A. Higgins, M. Imai, and T.E. Clarke, Jupiter's Io-C and Io-B decametric emission source morphology from LWA1 data analysis, in Planetary Radio Emissions VIII, edited by G. Fischer, G. Mann, M. Panchenko, and P. Zarka, Austrian Academy of Sciences Press, Vienna, 89-101, 2017.

Imai, M., A. Lecacheux, T. E. Clarke, C. A. Higgins, M. Panchenko, J. Dowell, K. Imai, A. I. Brazhenko, A. V. Frantsuzenko, and A. A. Konovalenko, The beaming structures of Jupiter's decametric common S-bursts observed from the LWA1, NDA, and URAN2 radio telescopes, Astrophys. J., 826, id. 176, 2016.

Konovalenko, A.A., et al. (71 co-authors), The modern radio astronomy network in Ukraine: UTR-2, URAN and GURT, Exp. Astron., 42, 11-48, 2016.

Koshida, T., T. Ono, M. Iizima, and A. Kumamoto, Jovian slow-drift shadow events J. Geophys. Res., 115, A01202, 2010.

Lecacheux, A., The Nançay Decameter Array: A useful step towards giant, new generation radio telescopes for long wavelength radio astronomy, in Radio Astronomy at Long Wavelengths, Geophys. Monogr., 119, p.321, 2000.

Melrose, D. B., R. G. Hewitt, and G. A. Dulk, Electron-cyclotron maser emission: Relative growth and damping rates for different modes and harmonics, J. Geophys. Res., 89, 897-904, 1984.

Oya, M., H. Oya, T. Ono, and M. Iizima, Analyses of Jovian decametric radiation S-bursts interacting with N-bursts, Earth Moon Planets, 88, 187-209, 2002.

Riihimaa, J. J., Structured events in the dynamic spectra of Jupiter's decametric radio emission, Astron. J., 73, 265-270, 1968.

Riihimaa, J. J., Bursts of type N in Jupiter's decametric radio spectra, Earth Moon Planets, 32, 9-19, 1985.

Riihimaa, J. J., and T.D. Carr, Interaction of S- and L-bursts in Jupiter's decametric radio spectra, Earth Moon Planets, 25, 373-387, 1981.

Taylor, G. B., et al. (34 co-authors), First light for the first station of the Long Wavelength Array, J. Astronom. Instrumentation, 1, id.1250004-284, 2012.

van Haarlem, M., et al. (200 co-authors), LOFAR: The LOw-Frequency ARray, Astron. Astrophys., 556, id.A2, 53 pp., 2013.

Wu, C. S., and L. C. Lee, A theory of terrestrial kilometric radiation, Astrophys. J., 230, 621-626, 1979.

Zarka, P., T. Farges, B. P. Ryabov, M. Abada-Simon, and L. Denis, A scenario for Jovian S-bursts, Geophys. Res. Lett., 23, 125-128, 1996. 